\title{
Toward the Undiscovered Dielectric Properties of Hybrid Acetamidinium Manganese Formate under High Pressure \\ Supplementary materials
}

\section{Table of contents}

1. Temperature and pressure evolution of $\varepsilon^{\prime}$ spectra

2. Calculations based on the equation of state

\section{Temperature and pressure evolution of $\varepsilon^{\prime}$ spectra}

The frequency dependent $\varepsilon^{\prime}$ spectra are characterized by a single step-like anomaly shifting towards higher frequencies with temperature increase, which is a characteristic feature for relaxation process. Moreover, as presented in Figure S1a, the static value of real part of dielectric permittivity, $\varepsilon_{\text {stat }}^{\prime}$, decreases gradually during heating at ambient pressure. The same phenomenon was observed while temperature lowering under isobaric high-pressure conditions, such as $250 \mathrm{MPa}$ (Figure S1b). The static value of real part of complex dielectric permittivity, $\varepsilon_{\text {stat }}^{\prime}$, experiences also a gradual decrease during compression at isothermal conditions, as presented for isotherm $298 \mathrm{~K}$ in Figure S2.
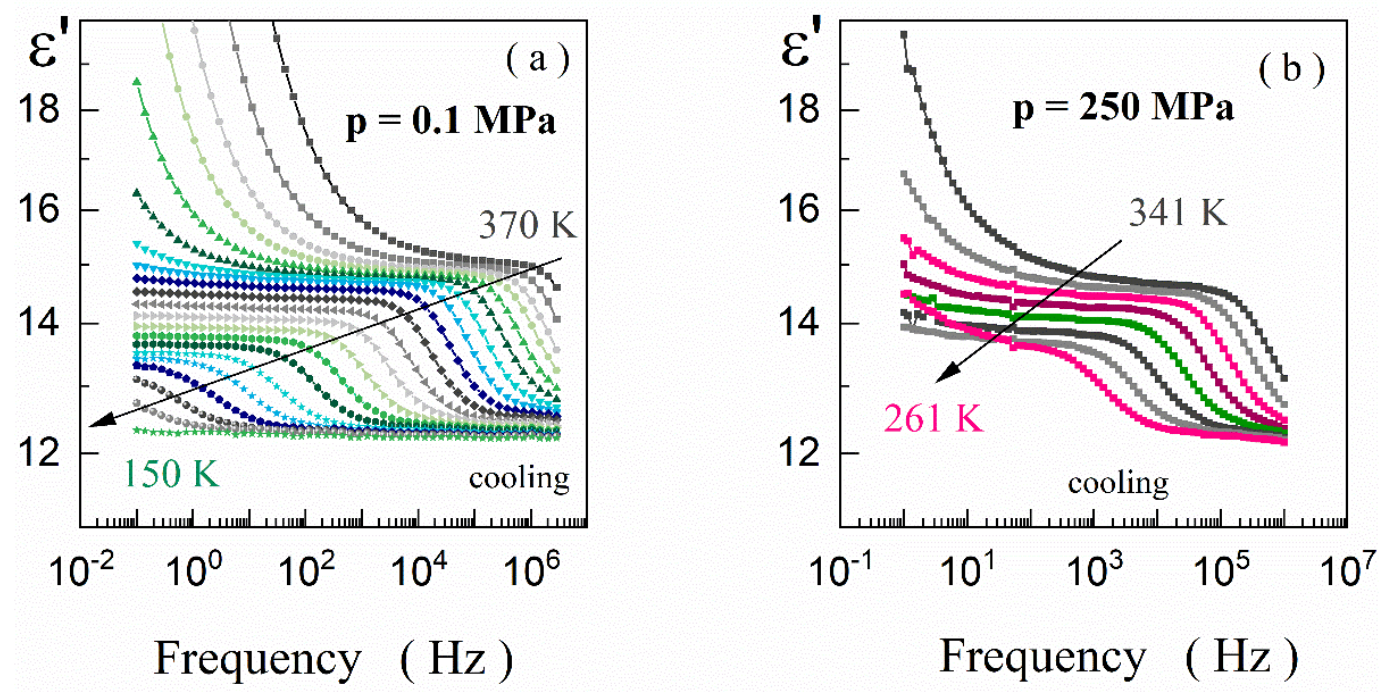

Figure S1. Frequency-dependent $\varepsilon^{\prime}$ spectra registered under isobaric conditions of (a) 0.1 $\mathrm{MPa}$ and (b) $250 \mathrm{MPa}$. 


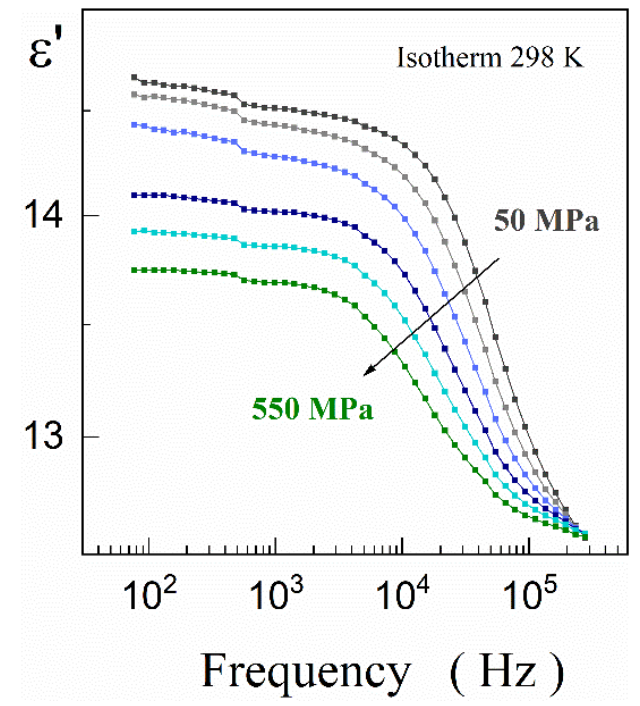

Figure S2. Frequency-dependent $\varepsilon^{\prime}$ spectra registered at $298 \mathrm{~K}$ during compression from 50 MPa up to $550 \mathrm{MPa}$.

\section{Calculations based on the equation of state}

To investigate the opposite effects of temperature and pressure elevation on the dynamics of $\mathrm{Ace}^{+}$cations, a temperature-pressure dependence for an isochronic conditions (that is for a constant relaxation time value) was derived from the determined equation of state:

$$
\tau(T, p)=\tau_{0} \exp \left(\frac{p \Delta V+E_{a}}{R T}\right)
$$

with temperature-independent activation volume: $\Delta V=6.4(2) \mathrm{cm}^{3} \mathrm{~mol}^{-1}$, activation energy: $E_{a}=0.54(1) \mathrm{eV}$ and logarithm of characteristic relaxation time: $\log _{10} \tau_{0}=-14.7(1)$. Herein, $2 \mu \mathrm{s}$ were taken as a relaxation time for the calculations. This relaxation time corresponds to the temperature-pressure conditions of phase transition for ambient pressure, what can be calculated from the equation (1) as following:

$$
\tau(302 K, 0.1 M P a)=2 \mu s
$$

The pressure-temperature conditions for the given relaxation time of $2 \mu$ s were calculated according to the formula:

$$
T=0.4342 \cdot \frac{p \cdot \Delta V+E_{a}}{R\left(\log _{10} \tau-\log _{10} \tau_{0}\right)}
$$

with $\Delta V, E_{a}$ and $\log _{10} \tau_{0}$ taken from the equation of state. As presented in Figure S3, for the selected relaxation time of $2 \mu$ s the $d T / d p$ parameter is equal to $37(2) \mathrm{K} / \mathrm{GPa}$, which means that 
compression of the compound up to $1 \mathrm{GPa}$ shifts the relaxation peak as much as lowering the temperature by $37 \mathrm{~K}$. Considering that the phase transition shifts with $d T_{0} / d p$ equal to 85(1) $\mathrm{K} / \mathrm{GPa}$, this figure also shows that the phase transition undergoes under non-isochronal conditions. It means that the external pressure exerts much less pronounced effect on the dynamics of reorientational motions of $\mathrm{Ace}^{+}$moieties than temperature lowering and, what is more, it acts to a different extent on acetamidinium cations and the cavern skeleton.

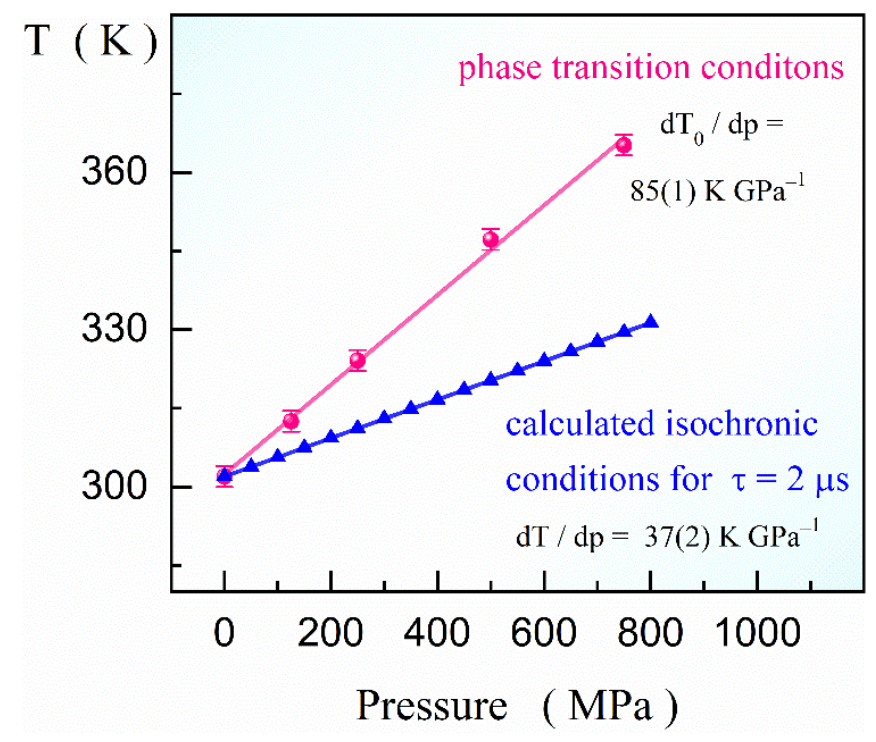

Figure S3. Comparison of calculated isochronic pressure-temperature conditions for $2 \mu \mathrm{s}$ with pressure-temperature dependence of phase transition for AceMn. 